\title{
DEPRESIÓN DEL ADULTO MAYOR PERUANO Y VARIABLES SOCIODEMOGRÁFICAS ASOCIADAS: ANÁLISIS DE ENDES 2017
}

\author{
DEPRESSION OF THE PERUVIAN ELDERLY ADULT AND ASSOCIATED SOCIODEMOGRAPHICAL \\ VARIABLES, ANALYSIS OF ENDES 2017 \\ Paola Baldeón-Martínez ${ }^{1, a}$, Consuelo Luna-Muñoz ${ }^{2, a, b}$, Sandra Mendoza-Cernaqué ${ }^{\text {,a }}$, Lucy E. Correa-López²
}

\begin{abstract}
RESUMEN
Objetivo: Determinar las variables sociodemográficas asociadas a la depresión en el adulto mayor peruano a partir de la información obtenida mediante la Encuesta Demográfica y de Salud Familiar (ENDES) del año 2017 realizada por el Instituto Nacional de Estadística e Informática (INEI). Métodos: Estudio observacional, correlacional y de corte transversal que usó una muestra probabilística de 4917 adultos mayores (60 años a más). Para la determinación de depresión en la población se usó como instrumento el Patient Health Questionnaire (PHQ) de 9 preguntas, incluido en el cuestionario de salud en la sección de salud mental del ENDES 2017. Resultados: La prevalencia de depresión en el adulto mayor peruano en el año 2017 fue de $14,2 \%$. Como variables sociodemográficas asociadas a la depresión fueron el sexo femenino [ORaj 1,995 $(1,684-2,364)$ ]; carecer de instrucción [ORaj 2,524 (2,126 - 2,996)]; la edad mayor de 75 años [ORaj 1,763 $(1,494-2,080)$ ]; vivir en una zona rural [ORaj 1,410 (1,198 -1,659)]; y ser pobre [ORaj 1,456 (1,229-1,724)]. No hubo asociación entre depresión y discapacidad $(p=0,704)$. [ORaj $1,103(0,663-1,835)$ ]. Conclusión: La prevalencia de depresión en el adulto mayor peruano es alta. Las variables sociodemográficas que estuvieron asociadas como factores de riesgo para el desarrollo de depresión en el adulto mayor fueron de sexo femenino, el carecer de instrucción, edad mayor de 75 años, vivir en zona rural y ser pobre.
\end{abstract}

Palabras clave: Adulto mayor; Depresión; Cuestionario PHQ-9; Factores de riesgo; Perú. (fuente: DeCS BIREME)

\section{ABSTRACT}

Objective: To determine the sociodemographic variables associated with depression in the elderly of Peru based on the information obtained through the Demographic and Family Health Survey (ENDES) of the year 2017 carried out by the National Institute of Statistics and Informatics (INEI). Methods: An observational, correlational and cross-sectional study that used a probabilistic sample of 4917 older adults (60 years and over). For the determination of depression in the population, the Patient Health Questionnaire (PHQ) of 9 questions included in the health questionnaire in the mental health section of ENDES 2017 was used as an instrument. Results: The prevalence of depression in the Peruvian elderly in 2017 was $14.2 \%$. As sociodemographic variables associated with depression were the female sex [OR 1.995 (1.684-2.364)]; lack instruction [ORaj 2.524 (2.126-2996)]; the age over 75 years [ORaj 1.763 (1.494 2.080)]; live in a rural area [ORaj 1.410 (1.198 $-1.659)$ ]; and be poor [ORaj $1.456(1.229-1.724)$ ]. It was not associated with disability $(p=0.704)$. [ORaj 1.103 (0.663-1835)]. Conclusion: It was concluded that the prevalence of depression in the Peruvian adult is high. Among the sociodemographic variables that were identified as risk factors for presenting depression were female sex, lacking education, age over 75 , living in rural areas and being poor.

Key words: Elderly; Depression; PHQ-9 Questionnaire; Risk factors; Perú. (source: MeSH NLM)

\footnotetext{
'Universidad Ricardo Palma, Lima-Perú.

${ }^{2}$ Instituto de Investigación en Ciencias Biomédicas, Universidad Ricardo Palma, Lima-Perú.

a Médica cirujana.

Doctora en Salud Pública, especialista en pediatría.
}

Citar como: Paola Baldeón-Martínez, Consuelo Luna-Muñoz, Sandra Mendoza-Cernaqué, Lucy E. Correa-López. Depresión del adulto mayor peruano y variables sociodemográficas asociadas: análisis de endes 2017. Rev. Fac. Med. Hum. Octubre 2019; 19(4):47-52. DOI 10.25176/RFMH.v19i4.2339 


\section{INTRODUCCIÓN}

En la actualidad la pirámide poblacional mundial está atravesando un cambio profundo. La transición demográfica y el incremento en la esperanza de vida han favorecido el aumento de la población de más de 60 años en el plano mundial, entre los principales: América Latina ${ }^{1,2}$.

Según proyecciones demográficas de la Organización Mundial de la Salud (OMS) a nivel mundial, el número de personas de 60 años o más está creciendo más rápidamente que cualquier otro grupo etario y que, para el año 2050, el $80 \%$ de las personas mayores habitará en países en desarrollo². Según los últimos censos poblacionales, el proceso de transición demográfica en el Perú, América Latina y el mundo muestra un incremento progresivo del grupo de personas mayores de 60 años $^{3}$. El Instituto Nacional de Estadística e Informática (INEI) afirma que en nuestro país hay más de 3 millones de adultos mayores, y para el año 2050 se estima que los adultos mayores representarán 8,7 millones de peruanos ${ }^{4}$. Sin embargo, este proceso de envejecimiento puede verse afectado negativamente por discapacidades físicas y mentales, una de las más notables es la depresión. Esta patología causa deterioro del funcionamiento de la vida diaria $y$ es poco diagnosticada y tratada en la atención primaria; además, aumenta la percepción de mala salud y la utilización de servicios de salud 5 .

Las autoridades han hecho énfasis en la identificación de este problema de salud pública, por lo que, en los últimos años, el Patient Health Questionnaire (PHQ), ha sido agregado por el Instituto Nacional de Estadística e Informática (INEI) en la Encuesta Demográfica y de Salud Familiar anual. Este instrumento es una herramienta de autoreporte con la posibilidad de ser usado en centros de atención primaria, ya que es un cuestionario breve y de gran ayuda para la investigación ${ }^{6}$. Todo ello contribuye no solo a identificar esta patología sino a las variables que giran en torno a ella, prevenibles o modificables, y elaborar políticas integrales y eficaces.

Debido a la necesidad de estudiar este importante fenómeno, es que este trabajo tiene el objetivo de determinar las variables sociodemográficas asociadas a la depresión en el adulto mayor según el análisis de ENDES 2017.

\section{MÉTODOS}

Se realizó un estudio de tipo observacional, de corte transcersal y correlacional a partir de los datos recogidos por la Encuesta Demográfica y de Salud Familiar del 2017 (ENDES 2017).

La población del presente estudio estuvo conformada por todos los adultos mayores del Perú con 60 a más años de edad durante el año 2017. La muestra que realizó el INEI fue bietápica, probabilística, estratificada y de tipo autoponderada, a nivel departamental y por tipo de área. El marco muestral lo constituye la información estadística y cartográfica proveniente de los Censos Nacionales XI de Población y VI de Vivienda del año 2007 y la Actualización SISFOH 2012-2013, y el material cartográfico actualizado para tal fin en el proceso de actualización cartográfica realizado para la ejecución de la ENDES. Las unidades de muestreo en el área urbana fueron por conglomerado y vivienda particular; y en el área rural, por Área de empadronamiento rural y vivienda particular?

Para identificar la variable dependiente (depresión) se utilizó el instrumento Patient Health Questionnaire (PHQ) que se encuentra en la pregunta 700 del cuestionario de salud de ENDES 2017, que a su vez contiene 9 sub preguntas, en la cual el valor de cada una de ellas era de 0 a 3 puntos, sumando una puntuación final que va de 0 a 27 puntos. Para la interpretación de resultados, definida por la puntuación final ${ }^{8}$, mayor o igual a 10 puntos significa depresión. La validación de la versión peruana del PHQ-9 se realizó en 2012 por especialistas en salud mental del Ministerio de Salud ${ }^{6}$.

Las principales variables fueron el sexo, la edad, el nivel de instrucción, la pobreza y la discapacidad. La edad se reagrupó en dos: 60 a 75 años y más de 75 . El nivel de instrucción se dicotomizó basado en la ausencia o presencia de algún grado de instrucción (inicial, primaria, secundaria o superior), al igual que la variable discapacidad (sí vs no). La variable pobreza se estableció en base a los quintiles, de los cuales el primero y el segundo fueron considerados pobres. El programa utilizado para el análisis estadístico fue Statistical Package for Social Sciences (SPSS), versión 21, empleando el módulo de muestras complejas del programa de acuerdo con los estratos, conglomerados y factores de ponderación de ENDES 2017. Se realizó un análisis univariado de los datos mediante la determinación de las frecuencias y porcentajes. Posteriormente, en la fase analítica, se utilizó el análisis bivariado y multivariado por regresión logística para evaluar el nivel de significancia estadística $(p<0,05)$. Las figuras fueron realizadas en Microsoft Excel 2016.

Se obtuvo la aprobación del Consejo de Facultad de la Universidad Ricardo Palma. El estudio estuvo bajo los lineamientos éticos para la investigación 
en salud propuestos en la Declaración de Helsinki y los entrevistadores se encargaron de obtener el consentimiento informado antes de aplicar la ENDES a los participantes. Este estudio se realizó bajo el contexto del Curso Taller de Titulación por Tesis de la Universidad Ricardo Palma?.

\section{RESULTADOS}

La población de estudio estuvo constituida por 4917 adultos mayores, entre quienes la prevalencia de depresión fue de 14,2\% (Figura 1).

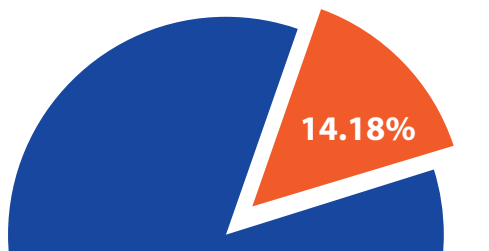

$85.82 \%$

Figura 1. Depresión en adultos mayores peruanos.

El sexo predominante fue el femenino, tanto en el grupo con depresión $(67,6 \%)$ como en el grupo sin depresión (51,1\%). Además, el 40,5\% de adultos mayores de 75 años tuvieron depresión. Más de la tercera parte de los adultos mayores con depresión $(37,4 \%)$ no tenían algún grado de instrucción. Por otro lado, el 42,6\% de los adultos mayores con depresión

residían en zonas rurales. Más aún, dos tercios de los adultos mayores con depresión vivían en condiciones de pobreza $(66,9 \%)$, y la discapacidad estuvo presente en menos del $3 \%$ de los adultos mayores con depresión y sin ella. En la Tabla 1 se vierten los datos descriptivos de las variables sociodemográficas que se incluyeron en el estudio.

Tabla1. Características sociodemográficas y depresión en los adultos mayores peruanos.

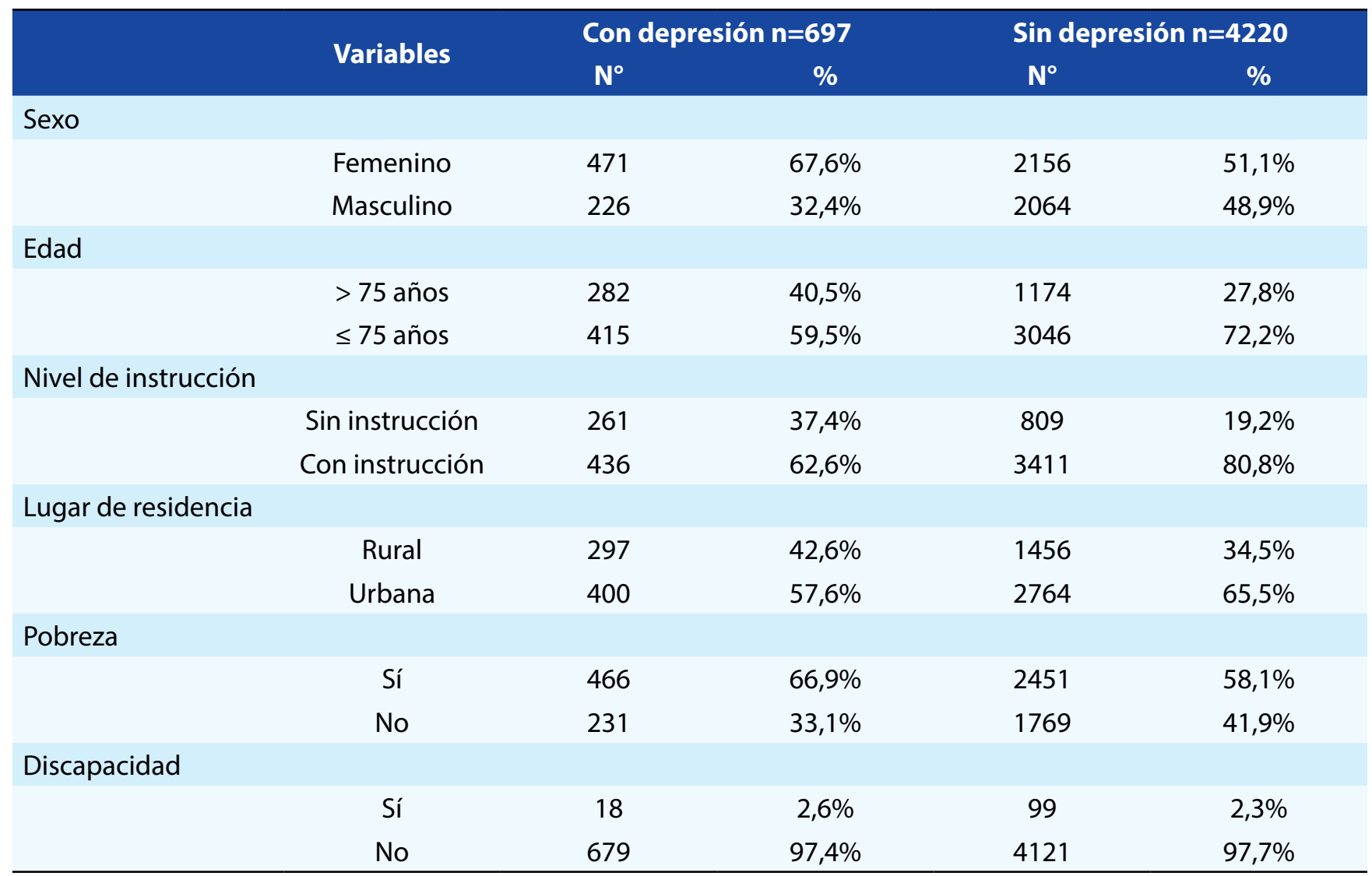


Tabla2. Análisis inferencial entre las características sociodemográficas y la depresión en el adulto mayor peruano.

\begin{tabular}{|c|c|c|c|c|}
\hline Factores & OR & IC $95 \%$ & p valor & OR (IC 95\%) \\
\hline \multicolumn{5}{|l|}{ Análisis bivariado } \\
\hline Sexo (femenino) & 1,995 & $1,684-2,364$ & 0,000 & $\mapsto$ \\
\hline Edad (>75 años vs $\leq 75$ años) & 1,763 & $1,494-2,080$ & 0,000 & $\mapsto-1$ \\
\hline $\begin{array}{l}\text { Nivel de instrucción (sin instrucción vs con } \\
\text { instrucción) }\end{array}$ & 2,524 & $2,126-2,996$ & 0,000 & $\longmapsto$ \\
\hline Lugar de residencia (rural vs urbana) & 1,410 & $1,198-1,659$ & 0,000 & $\mapsto-1$ \\
\hline Pobreza (sí vs no) & 1,456 & $1,229-1,724$ & 0,000 & $\mapsto-1$ \\
\hline Discapacidad (sí vs no) & 1,103 & $0,663-1,835$ & 0,704 & \\
\hline \multicolumn{5}{|l|}{ Análisis multivariado } \\
\hline Sexo (femenino) & 1,607 & $1,340-1,927$ & 0,000 & $H-1$ \\
\hline Edad (>75 años vs $\leq 75$ años) & 1,506 & $1,267-1,790$ & 0,000 & $H$ \\
\hline $\begin{array}{l}\text { Nivel de instrucción (sin instrucción vs con } \\
\text { instrucción) }\end{array}$ & 1,908 & $1,581-2,302$ & 0,000 & $\mapsto-1$ \\
\hline Lugar de residencia (rural vs urbana) & 1,238 & $1,014-1,511$ & 0,036 & 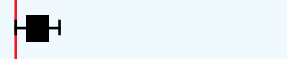 \\
\hline Pobreza (sí vs no) & 1,235 & $1,005-1,518$ & 0,045 & 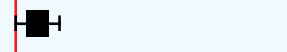 \\
\hline & & & ${ }_{0}^{L}$ & $\frac{1}{2}$ \\
\hline
\end{tabular}

En el análisis bivariado, los factores sociodemográficos asociados a depresión fueron el sexo femenino, la edad mayor a 75 años, la ausencia de nivel de instrucción, el lugar de residencia rural y pobreza. No hubo asociación estadísticamente significativa entre depresión y discapacidad (Tabla 2).

En el análisis multivariado continuaron siendo significativas. El sexo femenino presentaba asociación significativa de depresión (ORaj=1,607, IC 95\%=1,340$1,927)$ frente a los controles. La edad mayor a 75 años (ORaj=1,506, IC 95\%=1,267-1,790), presentaba mayor probabilidad de tener depresión en comparación con los controles. Además, no tener instrucción aumentaba el riesgo de padecer depresión (ORaj=1,908, IC $95 \%=1,581-2,302)$ frente a los controles. El lugar de residencia rural (ORaj=1,238, IC 95\%=1,0141,511) consignaba 1,2 veces la probabilidad de tener depresión. Por último, la probabilidad de tener depresión por la pobreza fue casi 1,2 veces las probabilidades de los controles (ORaj=1,235, IC $95 \%=1,005-1,518$ ) (Figura 2).

\section{DISCUSIÓN}

La salud mental en el adulto mayor puede ser vulnerada debido al maltrato: físico, sexual, psicológico, emocional, económico o material; y también por el abandono, falta de atención y respeto; los cuales pueden influenciar negativamente en el desarrollo de enfermedades mentales. Uno de los trastornos mentales que es frecuente entre los adultos mayores y que genera mayor padecimiento es la depresión, por lo que es esencial identificar los factores que contribuyen al desarrollo de tal enfermedad a fin de intervenir sobre ellos.

La prevalencia de depresión en la población de estudio fue de $14,2 \%$, lo cual fue semejante a la prevalencia obtenida por Martha Martina Chávez según la ENDES 2014-2015 con un valor cercano al $14,3 \%{ }^{8}$, esto quiere decir que no hubo aumento ni reducción en este periodo de tres años.

En nuestro estudio se buscó identificar las variables sociodemográficas asociadas a la depresión en 
el adulto mayor peruano basado en ENDES del año 2017, que además tiene carácter inferencial a la población nacional. Los factores asociados fueron el sexo femenino, la edad mayor de 75 años, ausencia de instrucción, lugar de residencia rural y la pobreza. Dichas variables han sido estudiadas por otros investigadores que concuerdan en su carácter determinante para el desarrollo de depresión en el adulto mayor.

$\mathrm{Paz}^{10}$. en su trabajo realizado en Lima Metropolitana y Callao encontró al sexo femenino como factor de riesgo asociado al episodio depresivo con un OR de 1,8, valor semejante al obtenido por Mirkena en Etiopía (OR: 1,72) ${ }^{11}$, y que coinciden con los resultados obtenidos en nuestro estudio (ORaj: 1,995). Esto tal vez se deba a mayor exposición de la mujer al maltrato psicológico, físico o sexual respecto al sexo masculino.

Respecto a la edad, el tener más de 75 años estuvo signifitivamente asociado al desarrollo de depresión. Martina $^{8}$ obtuvo resultados parecidos en una población de estudio semejante (ORaj: 1,556). Los adultos mayores han presenciado la pérdida de varios de sus familiares y amigos, así mismo este grupo etario presenta muchas comorbilidades, lo cual podría explicar las frecuentes manifestaciones depresivas en mayores de 75 años ${ }^{2}$.

El nivel de instrucción educativo también estuvo asociada con la depresión con un OR de 2,524, y acorde con el resultado del estudio de SeguraCardona, ${ }^{12}$ donde la menor escolaridad aumentaba la probabilidad de desarrollar depresión. El autor sostuvo que del grado de instrucción dependían la autopercepción de la salud así como el autocuidado en los adultos mayores.

El presente estudio demostró que la pobreza estuvo asociada al desarrollo de depresión en personas de la tercera edad, a la misma conclusión llegó el ecuatoriano Gómez ${ }^{13}$, ya que consideraba al nivel socioeconómico bajo como uno de los principales factores de riesgo que ocasionan depresión en las personas adultas mayores. Además, Paredes-Arturo ${ }^{14}$, en un estudio realizado en Colombia, encuentra asociación entre la depresión y el no tener ingresos económicos propios con un: OR: 3,713. Este resultado también lo observa
Bartwal en La India ${ }^{14}$. Vivir en condiciones de pobreza suele relacionarse a una menor accesibilidad a la salud y menor conocimiento de los síntomas de esta enfermedad mental.

Se evidenció que la discapacidad era poco prevalente en la población estudiada, además, no se pudo establecer relación entre la discapacidad y la depresión en los adultos mayores ( $p=0.704)$, lo cual difiere con el hallazgo de Martina ${ }^{8}$, quien obtuvo un OR de 2,63.

La principal limitación del estudio fue el uso de fuentes secundarias. Además, la Encuesta Demográfica y de Salud Familiar (ENDES) no fue elaborada específicamente para llegar a diagnósticos de depresión y menos aún para ser dirigida a los adultos mayores que son nuestra población de estudio. No obstante, podemos contar con que la información recopilada tiene validez por su calidad estadística que siguió un muestreo riguroso para la selección de la población de estudio y porque fue llevada a cabo por personal previamente capacitado.

\section{CONCLUSIÓN}

Los factores sociodemográficos asociados a depresión del adulto mayor peruano fueron el sexo femenino, la edad mayor o igual a 75 años, el lugar de residencia rural, no contar con instrucción y la pobreza.

Contribuciones de autoría: Las autoras participaron en la génesis de la idea, diseño de proyecto, recolección e interpretación de datos, análisis de resultados, preparación del manuscrito.

Financiamiento: Autofinanciado.

Conflicto de interés: Las autoras declaran no tener conflicto de interés en la publicación de este artículo.

Recibido: 01 junio del 2019

Aprobado: 30 julio del 2019

Correspondencia: Consuelo Luna Muñoz

Dirección: Instituto de Investigación en Ciencias Biomédicas (INICIB) Universidad Ricardo Palma, Av. Benavides 5440, Lima Perú

Teléfono: (01) 7080000

Correo:luna_consuelo@yahoo.es 


\section{REFERENCIAS BIBLIOGRÁFICAS}

1. Comisión Económica para América Latina y el Caribe (CEPAL) Envejecimiento, personas mayores y Agenda 2030 para el Desarrollo Sostenible: Perspectiva regional y de derechos humanos. Huenchan S, editor. Santiago: Naciones Unidas; 2018. 259 p.

2. World Health Organization. Envejecimiento y salud. WHO [Internet]. e 5 de febrero de 2018; Disponible en: https://www.who.int/news-room/ fact-sheets/detail/ageing-and-health

3. Varela-Pinedo L, Chávez-Jimeno $\mathrm{H}$, Tello-Rodríguez T, Ortiz-Saavedra P, Gálvez-Cano M, Casas-Vasquez P, et al. Perfil clínico, funcional y sociofamiliar del adulto mayor de la comunidad en un distrito de Lima, Perú. Rev Peru Med Exp Salud Publica [Internet]. el 5 de diciembre de 2015 [citado el 1 de septiembre de 2019];32(4):709. Disponible en: https://rpmesp.ins.gob.pe/index.php/rpmesp/article/view/1762

4. Instituto Nacional de Estadística e Informática. En el Perú viven más de 3 millones de adultos mayores. INEI [Internet]. el 25 de agosto de 2015 Disponible en: https://www.inei.gob.pe/prensa/noticias/en-el-peruviven-mas-de-3-millones-de-adultos-mayores-8570/

5. World Health Organization. Salud mental de adultos mayores. WHO [Internet]. el 12 de diciembre de 2017 [citado el 1 de septiembre de 2019]; Disponible en: https://www.who.int/news-room/fact-sheets/detail/ mental-health-of-older-adults

6. Calderón M, Gálvez-Buccollini JA, Cueva G, Ordoñez C, Bromley C, Fiestas F. Validación de la versión peruana del PHQ-9 para el diagnóstico de depresión. Rev Peru Med Exp Salud Publica [Internet]. diciembre de 2012 [citado el 1 de septiembre de 2019];29(4):578-9. Disponible en http://www.scielosp.org/scielo.php?script=sci_arttext\&pid=S172646342012000400027\&lng=es\&nrm=iso\&tlng=es

7. Instituto Nacional de Estadística e Informática. Series anuales de principales indicadores de la ENDES 1986-2017 [Internet]. Lima; 2018 [citado el 2 de septiembre de 2019]. Disponible en: https://www.inei.gob. pe/media/MenuRecursivo/publicaciones_digitales/Est/Lib1643/libro.pdf

8. Martina M, Ara MA, Gutiérrez C, Nolberto V, Piscoya J. Depresión y factores asociados en la población peruana adulta mayor según la ENDES 2014-2015. An la Fac Med [Internet]. el 23 de marzo de 2018 [citado el 1 de septiembre de 2019];78(4):393. Disponible en: http:// revistasinvestigacion.unmsm.edu.pe/index.php/anales/article/ view/14259
9. De La Cruz-Vargas JA, Correa-Lopez LE, Alatrista-Gutierrez de Bambaren M del S, Sanchez Carlessi HH, Luna Muñoz C, Loo Valverde $M$, et al. Promoviendo la investigación en estudiantes de Medicina y elevando la producción científica en las universidades: experiencia del Curso Taller de Titulación por Tesis. Educ Médica. julio de 2019;20(4):199-205.

10. Paz V. Factores Asociados al Episodio Depresivo en los Adultos Mayores de Lima Metropolitana y Callao. Rev An SALUD Ment [Internet]. el 15 de junio de 2016 [citado el 1 de septiembre de 2019];31(1). Disponible en: http://www.insm.gob.pe/ojsinsm/index.php/Revista1/ article/view/9

11. Mirkena Y, Reta MM, Haile K, Nassir Z, Sisay MM. Prevalence of depression and associated factors among older adults at ambo town, Oromia region, Ethiopia. BMC Psychiatry [Internet]. 2018 [citado el 2 de septiembre de 2019];18(1):338. Disponible en: http://www.ncbi.nlm. nih.gov/pubmed/30336773

12. Segura Cardona A, Cardona Arango D, Segura Cardona Á, Garzón Duque $M$, Duque MG. Riesgo de depresión y factores asociados en adultos mayores. Antioquia, Colombia. 2012. Rev Salud Pública [Internet]. el 15 de octubre de 2015 [citado el 1 de septiembre de 2019];17(2):184-94. Disponible en: http://www.revistas.unal.edu.co/ index.php/revsaludpublica/article/view/41295

13. Gómez S, Vinicio M. Factores que influyen en la depresión en los adultos mayores que acuden a los Centros Gerontológicos de la parroquia de Vilcabamba y Malacatos en el año 2016. Tesis de pregrado. [Ecuador]: Universidad Nacional de Loja; 2017.

14. Paredes-Arturo YV, Aguirre-Acevedo DC. Síntomas depresivos y factores asociados en población adulto mayor. Rev Mex Neurocienc [Internet]. el 15 de noviembre de 2016 [citado el 1 de septiembre de 2019];17(3):26-38. Disponible en: https://www.medigraphic.com/cgi$\mathrm{bin} / \mathrm{new} /$ resumen.cgi?IDARTICULO $=68923$

15. Bartwal J, Rawat C, Awasthi S. Rate of Depressive Symptoms and Associated Risk Factors Among the Elderly in Haldwani Block of Nainital District, Uttarakhand, India. Int J Nutr Pharmacol Neurol Dis [Internet]. 2017 [citado el 2 de septiembre de 2019];7(2):34. Disponible en: http:// www.ijnpnd.com/text.asp?2017/7/2/34/205288
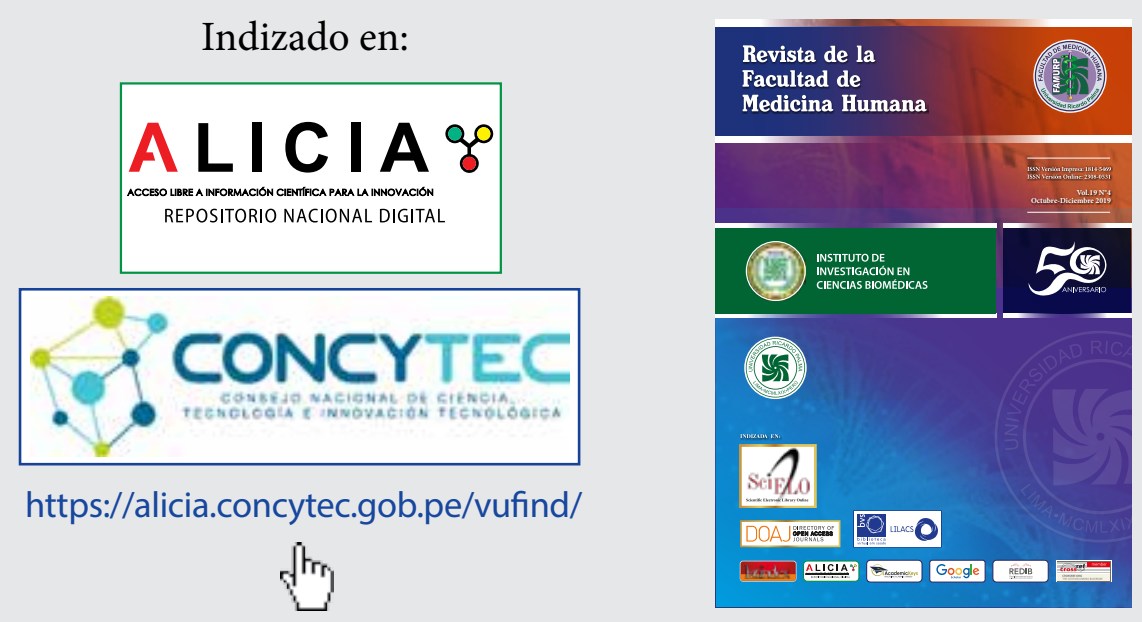